\title{
Dynamic Contrast-Enhanced Ultrasound of Gastric Cancer: Correlation with Perfusion CT and Histopathology
}

\author{
Ijin Joo, $M D^{1}$, Se Hyung Kim, $M D^{1}$, Dong Ho Lee, $M D^{1}$, Joon Koo Han, $M D^{1,2}$ \\ ${ }^{1}$ Department of Radiology, Seoul National University Hospital, Seoul National University College of Medicine, Seoul, Korea; ${ }^{2}$ Institute of Radiation \\ Medicine, Seoul National University Hospital, Seoul, Korea
}

Objective: To assess the relationship between contrast-enhanced ultrasound (CEUS) parameters and perfusion CT (PCT) parameters of gastric cancers and their correlation with histologic features.

Materials and Methods: This prospective study was approved by our Institutional Review Board. We included 43 patients with pathologically-proven gastric cancers undergoing CEUS using SonoVue ${ }^{\circledR}$ (Bracco) and PCT on the same day. Correlation between the CEUS parameters (peak intensity [PI], area under the curve [AUC], rise time [RT] from $10 \%$ to $90 \%$ of PI, time to peak $\left[\mathrm{TTP}_{\mathrm{US}}\right]$, and mean transit time $\left[\mathrm{MTT}_{\mathrm{US}}\right]$ ) and PCT parameters (blood flow, blood volume, $\mathrm{TTP}_{\mathrm{CT}}, \mathrm{MTT}_{\mathrm{CT}}$, and permeability surface product) of gastric cancers were analyzed using Spearman's rank correlation test. In cases of surgical resection, the CEUS and PCT parameters were compared according to histologic features using Mann-Whitney test.

Results: CEUS studies were of diagnostic quality in $88.4 \%$ (38/43) of patients. Among the CEUS parameters of gastric cancers, RT and $\mathrm{TTP}_{\text {US }}$ showed significant positive correlations with $\operatorname{TTP}_{\text {Ст }}$ (rho $=0.327$ and $0.374, p=0.045$ and 0.021 , respectively); PI and AUC were significantly higher in well-differentiated or moderately-differentiated tumors $(n=4)$ than poorlydifferentiated tumors $(n=18)\left(p=0.026\right.$ and 0.033 , respectively), whereas MTT $_{C T}$ showed significant differences according to histologic types (poorly cohesive carcinoma [PCC] vs. non-PCC), T-staging ( $\leq \mathrm{T} 2$ vs. $\geq \mathrm{T} 3$ ), N-staging (N0 vs. N-positive), and epidermal growth factor receptor expression ( $\leq$ faint vs. $\geq$ moderate staining) ( $p$ values $<0.05$ ).

Conclusion: In patients with gastric cancers, CEUS is technically feasible for the quantification of tumor perfusion and may provide correlative and complementary information to that of PCT, which may allow prediction of histologic features.

Keywords: Stomach cancer; Perfusion imaging; Contrast-enhanced ultrasound (CEUS); Histologic grade

\section{INTRODUCTION}

In patients with gastric cancer, surgical resection is the gold standard for curative treatment. However, gastric cancers are often diagnosed at an advanced stage, leading to poor prognosis $(1,2)$. To improve the patients' outcomes, neoadjuvant, adjuvant, and palliative therapeutic options have been actively investigated in many clinical trials (1,
2). Accurate classification and risk stratification of patients with gastric cancers at initial diagnosis are important for making management decisions and predicting prognosis (3). The endoscopic biopsy specimen is used to determine the histologic type and grade of gastric cancer but has limitation to represent the entire tumor due to intratumoral heterogeneity; quantitative imaging analysis including a larger tumor area has potential to reflect the histologic

Received April 27, 2018; accepted after revision December 10, 2018.

This study was supported funding from the Basic Science Research Program through the National Research Foundation of Korea (NRF) funded by the Ministry of Science, ICT \& Future Planning, Republic of Korea (2016R1A2B4007762) and by Seoul National University Hospital Research Fund No. 03-2016-450.

Corresponding author: Se Hyung Kim, MD, Department of Radiology, Seoul National University Hospital, Seoul National University College of Medicine, 101 Daehak-ro, Jongno-gu, Seoul 03080, Korea.

- Tel: (822) 2072-2057• Fax: (822) 743-6385• E-mail: shkim7071@gmail.com

This is an Open Access article distributed under the terms of the Creative Commons Attribution Non-Commercial License (https:// creativecommons.org/licenses/by-nc/4.0) which permits unrestricted non-commercial use, distribution, and reproduction in any medium, provided the original work is properly cited. 
characteristics of the tumor more accurately $(4,5)$ and can be applied even in patients with unresectable gastric cancers.

Perfusion imaging techniques are utilized in many oncologic fields as they provide quantitative imaging biomarkers which reflect the tumor's microvascular structures and/or function (6-11). Perfusion imaging of the stomach has been considered as challenging due to peristaltic movement; however, due to recent advances in image registration and fast imaging technique, studies have shown the technical feasibility of perfusion CT (PCT) and dynamic contrast-enhanced (DCE) MRI for evaluating gastric cancers (12-14). In addition, reports have indicated that the tumor perfusion parameters of PCT or DCE-MRI differ significantly according to the tumor stage and histologic grade of gastric cancer $(12,14)$, and can be used as prognostic predictors of the tumor response to palliative chemotherapy (13). More recently, double contrast-enhanced ultrasound (CEUS) with the combined use of oral and intravenous ultrasound contrast agents has been introduced as a reliable diagnostic method to evaluate both the morphologic features and enhancement patterns of gastric cancers (15). Reports have indicated that CEUS is useful in the differential diagnosis $(15,16)$, preoperative gross classification (17), and T-staging (18) of gastric cancers. Moreover, the respiratory motion correction techniques in free-breathing CEUS (19) enable accurate quantification of the tumor perfusion of gastric cancers $(20,21)$.

While both CEUS and PCT can provide information related to tumor perfusion, CEUS has advantages over PCT in terms of safety profile, cost, and lack of ionizing radiation (22); hence if CEUS is confirmed as a suitable alternate to PCT for quantification of tumor perfusion, it may be beneficial especially to patients with renal dysfunction or pediatric patients, and those enrolled in clinical trials who undergo repeat monitoring of response.

This study aimed to assess the feasibility of quantitative DCE CEUS for use in patients with advanced gastric cancers and investigate the relationship between CEUS parameters and PCT parameters of gastric cancers as well as their correlation with histologic features.

\section{MATERIALS AND METHODS}

\section{Study Population}

This prospective study was approved by our Institutional Review Board. Written informed consent was obtained from each patient. This study population was a subgroup of a prospective study which primarily aimed to evaluate the performance of PCT in patients with gastric cancers. Inclusion criteria were as follows: 1) patients with biopsyproven gastric cancers staged as advanced gastric cancers (T2 or greater) through endoscopy scheduled to undergo baseline PCT who were enrolled in the main study; 2 ) those who provided informed consent to undergo CEUS for gastric cancers after undergoing PCT. Patients whose gastric cancers were not clearly visualized through B-mode ultrasound imaging before CEUS were excluded. From December 2015 to August 2016, among those patients scheduled to undergo baseline PCT for gastric cancers, 53 patients were assessed for eligibility using B-mode ultrasonography after ingesting $500-1000 \mathrm{~mL}$ of water by one of two radiologists with 5 and 15 years of experience in CEUS, respectively. Among these, 10 patients were excluded as their gastric cancers were not clearly visible on ultrasound imaging. Finally, 43 patients (23 male individuals and 20 female individuals) with mean ( \pm standard deviation) age of $61.4( \pm 15.0)$ years (age range, $19-85$ years) were enrolled in the study. Some PCT data from this study population $(n=19)$ were also included in the prior study to assess the performance of PCT for predicting histologic features (23). Different from the prior study (23), the current study aimed to evaluate the performance of CEUS and correlation between CEUS and PCT.

\section{PCT Protocol}

All PCT examinations were performed on a 64-slice multidetector CT scanner (SOMATOM Definition; Siemens Healthineers, Erlangen, Germany). Each patient was administered an intravenous injection of $10 \mathrm{mg}$ of hyoscine butylbromide (Buscopan ${ }^{\circledR}$; Boehringer Ingelheim Korea, Seoul, Korea) 5 minutes prior to the PCT study to minimize bowel movement, and $500-1000 \mathrm{~mL}$ of water immediately prior to the PCT study to distend the stomach. After acquisition of non-contrast images, the scan location for PCT was determined according to the site of the gastric cancer. A total of $40 \mathrm{~mL}$ of a non-ionic iodinated contrast agent (350 mgI/mL; iobitridol, Xenetix 350; Guerbet, Aulnay-sous-Bois, France) was administered at a rate of 5 $\mathrm{mL} / \mathrm{sec}$ followed by $50 \mathrm{~mL}$ of normal saline at the same rate using a power injector via the antecubital vein. Acquisition of PCT images was started 6 seconds after injection of the contrast agent and lasted for 60 seconds including 15 phases at 3.6-second interval. The following acquisition parameters were used: scan range of $8 \mathrm{~cm}$, rotation time of 0.4 seconds, effective tube current of $100 \mathrm{mAs}$ at $80 \mathrm{kVp}$, and slice thickness of $3 \mathrm{~mm}$. All patients were asked to 
breathe quietly during the PCT examination.

\section{CEUS Protocol}

CEUS examinations were performed on the same day of the PCT examinations using a single ultrasound scanner (LOGIQ E9, GE Healthcare, Milwaukee, WI, USA) with a convex array probe (1-6 MHz) by one of the two radiologists who also assessed the eligibility of the patients. Patients were requested to maintain nil per os status for at least 4 hours prior to the CEUS examination. After the patients had ingested 500-1000 $\mathrm{mL}$ of water, B-mode ultrasound was performed in supine or left lateral decubitus position, and the imaging plane showing the largest tumor dimension at parallel axis to the respiratoryrelated movement of the tumor was selected. All patients were requested to breathe quietly during the CEUS examination. CEUS images were obtained in contrast-specific ultrasound mode with the following parameters: harmonic mode, dynamic range of $69 \mathrm{~dB}$, mean gain of $24 \mathrm{~dB}$, mechanical index of $0.15-0.17$, and imaging frame rate of approximately 11 frames per second. Immediately after intravenous bolus administration of $2.4 \mathrm{~mL}$ of SonoVue ${ }^{\circledR}$ (Bracco, Milan, Italy) via the antecubital vein (18-guage or 20-gauge cannula) followed by $5-\mathrm{mL}$ normal saline, continuous scanning was conducted for 90 seconds without changing the position of the probe. The raw digital data were stored as cine loops and exported as Digital Imaging and Communications in Medicine files for further analysis.

\section{Image Analysis}

Post-processing of PCT and CEUS was performed by one radiologist who was blinded to the histologic features and operation records.

PCT analysis was performed using a post-processing platform with dedicated PCT software (Syngo Volume Perfusion CT body ${ }^{\circledR}$; Siemens Healthineers). First, motion correction using a non-rigid registration method was applied. Subsequently, the abdominal aorta was selected as the input artery, and perfusion analysis was performed using a combination of the maximum slope approach and delayed Patlak analysis. Through PCT software, a freehand region of interest (ROI) was drawn on the gastric cancer in the representative axial plane so as to include the largest tumor dimension, and blood flow (BF) ( $\mathrm{mL} / 100 \mathrm{~g} / \mathrm{min})$, blood volume (mL/100 g), time to peak (TTP $\left.\mathrm{TT}_{\mathrm{C}}\right)$ (second), mean transit time ( $\mathrm{MTT}_{\mathrm{CT}}$ ) (second), and permeability surface product $(\mathrm{mL} / 100 \mathrm{~g} / \mathrm{min})$ of the selected ROI were automatically calculated.
CEUS analysis was performed using a post-processing platform with dedicated CEUS software (VueBox ${ }^{\circledR} ;$ Bracco) (19). After applying automatic in-plane motion correction, a freehand ROI was drawn on the gastric cancer lesion. CEUS studies were determined to be of diagnostic image quality according to the quality of fit (QOF) provided by the software, which represents the degree of agreement between the raw data and fitted mathematical model. If the QOF was less than $50 \%$ in up to three attempts of placement of the ROI, the CEUS examination was regarded as non-diagnostic. The CEUS software then provided quantitative CEUS parameters of the selected ROI including the peak intensity (PI), area under the curve (AUC), rise time (RT) from $10 \%$ to $90 \%$ of the PI, $\mathrm{TTP}_{\mathrm{US}}$, and $\mathrm{MTT}_{\mathrm{US}}$ (Fig. 1).

\section{Histologic Analysis}

In all patients who underwent surgical resection of gastric cancers, the pathologic $\mathrm{T}$ and $\mathrm{N}$-stage according to the American Joint Committee on Cancer 7th edition classification, tumor differentiation status (welldifferentiated [WD], moderately-differentiated [MD], or poorly-differentiated [PD]) and histologic type (poorly cohesive carcinoma [PCC] or non-PCC) were reported. In immunohistochemistry analysis, the expression levels of epidermal growth factor receptor (EGFR) and human epidermal growth factor receptor 2 (HER-2) were scored as follows: 0 , no membrane staining; $1+$, faint or partial staining; $2+$, moderate staining; and $3+$, strong staining.

\section{Statistical Analysis}

Gastric cancers with technical success and those with

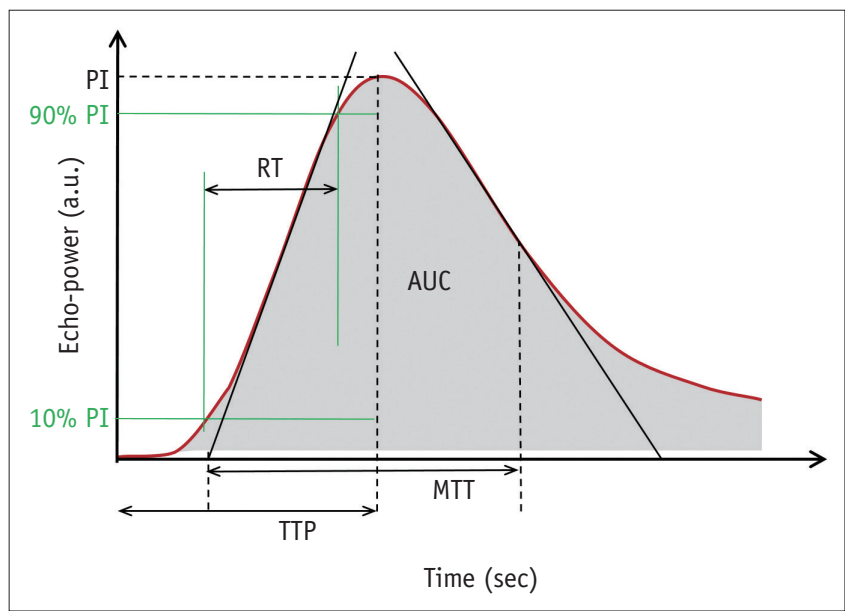

Fig. 1. Calculation of perfusion parameters using CEUS. $A U C=$ area under curve, CEUS = contrast-enhanced ultrasound, MTT = mean transit time, $\mathrm{PI}=$ peak intensity, $\mathrm{RT}=$ rise time, $\mathrm{TTP}=$ time to peak 
technical failure were compared with regards to the tumor location and Borrmann type based on the endoscopic reports. In all patients with CEUS images of diagnostic quality, the correlation between CEUS and PCT parameters of gastric cancers was analyzed using Spearman's rank correlation analysis. In addition, in patients who had undergone gastrectomy, the CEUS and PCT parameters were compared according to histologic features (T-staging, $\leq \mathrm{T} 2$ vs. $\geq \mathrm{T} 3$; N-staging, N0 vs. N-positive; tumor differentiation, WD or MD vs. PD; histologic type, PCC vs. non-PCC; EGFR or HER-2 expression, 0 or $1+$ vs. $2+$ or $3+$ ) using MannWhitney test. A $p$ value of less than 0.05 was considered as statistical significance. All statistical analyses were performed using commercially available statistical software (MedCalc ${ }^{\circledR}$, version 17.9.2; MedCalc Software, Ostend, Belgium).

\section{RESULTS}

Among the 43 patients included in this study, CEUS examinations of 38 patients $(88.4 \%)$ were classified as diagnostic quality for the assessment of gastric cancers indicating technical success of CEUS, while the other five patients $(11.6 \%)$ were classified as non-diagnostic quality indicating technical failure. The comparison of tumor characteristics of patients with technical success versus failure of CEUS is shown in Table 1. Gastric cancers at the upper location (cardia, fundus, or high body) showed a significantly higher technical failure rate than those at the lower location. Among the 38 patients with technical success, gastrectomy was performed in 22 patients and the interval between CEUS and surgery was $28.4 \pm 10.2$ days (mean \pm standard deviation) (range, 11-44 days). The detailed histologic features of gastric cancers in patients who underwent surgical resection are shown in Table 2.

\section{Correlation between CEUS and PCT Parameters}

Among the quantitative parameters of CEUS and PCT, the

Table 2. Histologic Features of Gastric Cancers in Patients Who Underwent Gastrectomy $(n=22)$

\begin{tabular}{|c|c|}
\hline Histologic Features & No. of Patients (\%) \\
\hline \multicolumn{2}{|l|}{ T-staging } \\
\hline $\mathrm{T} 1$ & $3(13.6)$ \\
\hline $\mathrm{T} 2$ & $4(18.2)$ \\
\hline T3 & $8(36.4)$ \\
\hline T4 & $7(31.8)$ \\
\hline \multicolumn{2}{|l|}{$\mathrm{N}$-staging } \\
\hline No & $6(27.3)$ \\
\hline N1 & $3(13.6)$ \\
\hline $\mathrm{N} 2$ & $6(27.3)$ \\
\hline N3 & $7(31.8)$ \\
\hline \multicolumn{2}{|l|}{ Tumor differentiation } \\
\hline WD & $1(4.5)$ \\
\hline MD & $3(13.6)$ \\
\hline PD & $18(81.8)$ \\
\hline \multicolumn{2}{|l|}{ Histologic types } \\
\hline Tubular adenocarcinoma & $12(54.5)$ \\
\hline Papillary adenocarcinoma & $1(4.5)$ \\
\hline PCC & $9(40.9)$ \\
\hline \multicolumn{2}{|l|}{ EGFR expression } \\
\hline 0 & $2(9.1)$ \\
\hline $1+$ & $3(13.6)$ \\
\hline $2+$ & $10(45.5)$ \\
\hline $3+$ & $7(31.8)$ \\
\hline \multicolumn{2}{|l|}{ HER-2 expression } \\
\hline 0 & $15(68.2)$ \\
\hline $1+$ & $3(13.6)$ \\
\hline $2+$ & $3(13.6)$ \\
\hline $3+$ & $1(4.5)$ \\
\hline
\end{tabular}

0 , no membrane staining; $1+$, faint or partial staining; $2+$, moderate staining; and $3+$, strong staining. EGFR = epidermal growth factor receptor, HER-2 = human epidermal growth factor receptor $2, M D=$ moderately-differentiated, $\mathrm{PCC}=$ poorly cohesive carcinoma, $\mathrm{PD}=$ poorly-differentiated, $\mathrm{WD}=$ well-differentiated

Table 1. Characteristics of Gastric Cancers in Patients with Technical Success and Failure on CEUS

\begin{tabular}{lcc}
\hline \multicolumn{1}{c}{ Endoscopic Findings of Gastric Cancers } & Technical Success $(\mathrm{n}=38)$ & Technical Failure $(\mathrm{n}=5)$ \\
\hline Tumor locations & & \\
Upper location & $7(18.4)$ & $3(60.0)$ \\
Lower location & $31(81.6)$ & $2(40.0)$ \\
Borrmann types & $2(5.3)$ & $1(20.0)$ \\
II & $34(89.5)$ & $4(80.0)$ \\
III & $2(5.3)$ & 0 \\
IV & & 0.041 \\
\hline
\end{tabular}

Numbers in parentheses are percentages. Lower location: involvement of low body to antrum regardless involvement of cardia/fundus/ high-mid body, Upper location: involvement of cardia, fundus, or high-mid body without involvement of low body-antrum. CEUS = contrast-enhanced ultrasound 
time-related parameters of RT and TTP US of CEUS showed significant positive correlations with $\operatorname{TTP}_{\mathrm{CT}}$ (rho $=0.327$ and $0.374, p=0.045$ and 0.021 , respectively); the other CEUS parameters showed no significant correlations with any PCT parameters (Table 3).

\section{Comparison of CEUS and PCT Parameters according to Histologic Features}

CEUS and PCT parameters according to the histologic features of gastric cancers in patients who underwent surgical resection are described in Tables 4 and 5, respectively.

Among the CEUS parameters, the amplitude-related parameters of PI and AUC showed significant differences according to the tumor differentiation status (WD/MD vs. PD: median PI, 1810 a.u. vs. 106 a.u., $p=0.026$; median AUC, 22100 a.u. vs. 1555 a.u., $p=0.033$, respectively) (Table 4). Representative examples are shown in Figures 2 and 3.

Table 3. Correlation between CEUS Parameters and PCT Parameters of Gastric Cancer

\begin{tabular}{ccccrr}
\hline \multirow{2}{*}{ CEUS Parameters } & \multicolumn{5}{c}{ PCT Parameters } \\
\cline { 2 - 6 } & BF & BV & TTP $_{\text {CT }}$ & MTT $_{\text {CT }}$ & PBS \\
\hline PI & $-0.099(0.552)$ & $-0.040(0.811)$ & $0.165(0.323)$ & $0.186(0.264)$ & $0.019(0.909)$ \\
AUC & $-0.067(0.688)$ & $-0.009(0.959)$ & $0.160(0.336)$ & $0.147(0.378)$ & $-0.031(0.854)$ \\
RT & $0.048(0.774)$ & $0.145(0.387)$ & $0.327^{*}(0.045)$ & $0.020(0.906)$ & $0.036(0.831)$ \\
TTP $_{\text {US }}$ & $0.105(0.532)$ & $0.210(0.205)$ & $0.374^{*}(0.021)$ & $0.094(0.575)$ & $0.011(0.947)$ \\
MTT US & $0.077(0.647)$ & $0.068(0.685)$ & $0.135(0.418)$ & $-0.022(0.898)$ & $-0.045(0.791)$ \\
\hline
\end{tabular}

Data are Spearman's correlation coefficients (rho). Numbers in parentheses are $p$ values. ${ }^{*} p<0.05$. AUC $=$ area under curve, BF $=$ blood flow, $\mathrm{BV}=$ blood volume, $\mathrm{MTT}=$ mean transit time, $\mathrm{PBS}=$ permeability surface product, $\mathrm{PCT}=$ perfusion $\mathrm{CT}, \mathrm{PI}=$ peak intensity, $\mathrm{RT}=$ rise time, TTP $=$ time to peak

Table 4. Comparison of CEUS Parameters according to Histologic Features of Gastric Cancer

\begin{tabular}{|c|c|c|c|c|c|}
\hline \multirow{2}{*}{ Histologic Features } & \multicolumn{5}{|c|}{ CEUS Parameters } \\
\hline & PI (a.u.) & AUC (a.u.) & $\mathrm{RT}(\mathrm{sec})$ & $\operatorname{TTP}_{\text {uS }}(\mathrm{sec})$ & $\mathrm{MTT}_{\text {US }}(\mathrm{sec})$ \\
\hline \multicolumn{6}{|l|}{ T-staging } \\
\hline$\leq \mathrm{T} 2(\mathrm{n}=7)$ & $153(39-911)$ & $5080(508-13625)$ & $8.6(6.5-9.6)$ & $13.1(9.2-14.8)$ & $99.0(17.6-152.8)$ \\
\hline$\geq T 3(n=15)$ & $354(64-1800)$ & 3470 (801-29825) & $6.4(5.7-8.3)$ & $9.9(7.6-13.9)$ & $25.1(22.0-39.1)$ \\
\hline$p$ value & 0.535 & 0.680 & 0.267 & 0.630 & 0.298 \\
\hline \multicolumn{6}{|l|}{$\mathrm{N}$-staging } \\
\hline NO $(n=6)$ & $842(153-1060)$ & $13150(5080-14300)$ & $7.9(6.5-9.7)$ & $12.0(9.4-15.1)$ & $23.7(15.3-111.6)$ \\
\hline$N$-positive $(n=16)$ & $106(45-1680)$ & $1555(548-29750)$ & $6.6(5.7-9.0)$ & $9.7(7.0-14.0)$ & $30.2(22.4-46.0)$ \\
\hline$p$ value & 0.407 & 0.329 & 0.329 & 0.407 & 0.693 \\
\hline \multicolumn{6}{|l|}{ Tumor differentiation } \\
\hline WD/MD $(n=4)$ & $1810(1020-2590)$ & $22100(14200-39350)$ & $6.9(6.4-7.9)$ & $10.2(9.4-12.0)$ & $22.8(14.8-98.5)$ \\
\hline$P D(n=18)$ & $106(37-734)$ & 1555 (427-12200) & $6.9(5.6-9.7)$ & $10.0(7.4-14.3)$ & $27.6(22.9-47.2)$ \\
\hline$p$ value & $0.026^{*}$ & $0.033^{*}$ & 1.000 & 1.000 & 0.594 \\
\hline \multicolumn{6}{|l|}{ Histologic type } \\
\hline Non-PCC $(n=13)$ & $75(33-1000)$ & $1200(329-14150)$ & $6.7(5.6-8.8)$ & $9.5(7.8-14.0)$ & $24.4(17.6-40.6)$ \\
\hline $\operatorname{PCC}(n=9)$ & $354(97-2533)$ & $5080(1613-33775)$ & $7.2(6.2-11.4)$ & $13.1(8.1-15.3)$ & $30.0(22.7-88.8)$ \\
\hline$p$ value & 0.110 & 0.144 & 0.556 & 0.556 & 0.393 \\
\hline \multicolumn{6}{|l|}{ EGFR expression } \\
\hline $0 / 1+(n=5)$ & $61(51-978)$ & $1440(943-10653)$ & $6.4(5.9-9.8)$ & $13.7(9.1-14.0)$ & $23.1(19.8-68.2)$ \\
\hline $2+/ 3+(n=17)$ & $704(66-1560)$ & $11500(608-29675)$ & $7.2(5.8-8.8)$ & $9.5(7.8-14.3)$ & $30.4(21.8-55.7)$ \\
\hline$p$ value & 0.649 & 0.543 & 0.940 & 0.704 & 0.543 \\
\hline \multicolumn{6}{|l|}{ HER-2 expression } \\
\hline $0 / 1+(n=18)$ & $254(61-1440)$ & $4275(668-29600)$ & $6.8(5.6-9.7)$ & $10.0(7.4-14.3)$ & $24.8(21.7-44.7)$ \\
\hline $2+/ 3+(n=4)$ & $559(33-1810)$ & $7730(644-22100)$ & $7.0(6.5-8.3)$ & $10.2(9.3-12.4)$ & $64.7(22.4-140.9)$ \\
\hline$p$ value & 0.837 & 0.774 & 0.902 & 0.967 & 0.434 \\
\hline
\end{tabular}

Data are median values. Numbers in parentheses indicate interquartile range. $p$ values were calculated using Mann-Whitney test. ${ }^{*} p<0.05$. 
Table 5. Comparison of PCT Parameters according to Histologic Features of Gastric Cancer

\begin{tabular}{|c|c|c|c|c|c|}
\hline \multirow{2}{*}{ Histologic Features } & \multicolumn{5}{|c|}{ PCT Parameters } \\
\hline & $\mathrm{BF}(\mathrm{mL} / 100 \mathrm{~g} / \mathrm{min})$ & BV $(\mathrm{mL} / 100 \mathrm{~g})$ & $\operatorname{TTP}_{\text {CT }}(\mathrm{sec})$ & $\mathrm{MTT}_{\mathrm{CT}}(\mathrm{sec})$ & PBS $(\mathrm{mL} / 100 \mathrm{~g} / \mathrm{min})$ \\
\hline \multicolumn{6}{|l|}{ T-staging } \\
\hline$\leq \mathrm{T} 2(\mathrm{n}=7)$ & $33.6(29.6-48.1)$ & $8.6(5.9-11.9)$ & $16.8(15.7-18.2)$ & $8.7(6.8-10.2)$ & $27.6(21.8-37.4)$ \\
\hline$\geq \mathrm{T} 3(n=15)$ & $50.6(40.9-62.0)$ & $10.6(9.5-16.1)$ & $15.7(14.7-18.1)$ & $11.2(9.9-13.3)$ & $41.8(21.3-48.7)$ \\
\hline$p$ value & 0.106 & 0.106 & 0.630 & $0.032^{*}$ & 0.237 \\
\hline \multicolumn{6}{|l|}{$\mathrm{N}$-staging } \\
\hline NO $(n=6)$ & $42.6(32.7-52.2)$ & $9.3(8.6-12.9)$ & $16.2(15.1-18.2)$ & $7.9(6.7-10.6)$ & $31.3(20.6-38.1)$ \\
\hline N-positive $(n=16)$ & $42.1(33.5-58.9)$ & $10.5(7.2-15.2)$ & $16.7(14.5-18.1)$ & $11.1(9.3-13.1)$ & $37.9(21.4-47.3)$ \\
\hline$p$ value & 0.914 & 0.641 & 0.693 & $0.049^{*}$ & 0.641 \\
\hline \multicolumn{6}{|l|}{ Tumor differentiation } \\
\hline WD/MD $(n=4)$ & $42.8(31.1-62.6)$ & $9.6(7.2-13.3)$ & $15.9(14.9-18.7)$ & $10.9(8.7-11.4)$ & $28.3(21.1-48.0)$ \\
\hline$P D(n=18)$ & $42.1(2.7-58.4)$ & $10.4(7.3-14.2)$ & $16.7(14.7-18.1)$ & $10.5(8.5-12.6)$ & $37.0(21.2-46.3)$ \\
\hline$p$ value & 1.000 & 0.774 & 1.000 & 0.837 & 0.967 \\
\hline \multicolumn{6}{|l|}{ Histologic type } \\
\hline Non-PCC $(n=13)$ & $51.9(31.8-60.2)$ & $9.9(6.9-16.4)$ & $15.7(14.9-17.5)$ & $9.0(7.0-11.3)$ & $25.3(18.2-41.5)$ \\
\hline $\mathrm{PCC}(n=9)$ & $41.5(32.6-50.8)$ & $10.5(8.3-12.6)$ & $18.1(14.2-19.9)$ & $12.5(10.6-14.9)$ & $41.8(35.7-48.4)$ \\
\hline$p$ value & 0.471 & 0.948 & 0.431 & $0.011^{*}$ & 0.082 \\
\hline \multicolumn{6}{|l|}{ EGFR expression } \\
\hline $0 / 1+(n=5)$ & $40.9(35.5-45.8)$ & $10.3(6.9-12.9)$ & $18.0(16.7-18.6)$ & $13.6(10.9-15.6)$ & $35.9(24.2-42.0)$ \\
\hline $2+/ 3+(n=17)$ & $50.6(31.8-60.2)$ & $10.5(8.2-16.0)$ & $15.7(14.7-18.2)$ & $10.5(7.4-11.3)$ & $35.0(20.1-46.9)$ \\
\hline$p$ value & 0.595 & 0.649 & 0.493 & $0.031^{*}$ & 1.000 \\
\hline \multicolumn{6}{|l|}{ HER-2 expression } \\
\hline $0 / 1+(n=18)$ & $41.3(32.7-52.2)$ & $10.1(7.3-12.9)$ & $17.0(14.7-18.2)$ & $10.5(8.5-12.6)$ & $35.5(21.2-46.3)$ \\
\hline $2+/ 3+(n=4)$ & $55.2(36.9-65.9)$ & $12.4(8.4-15.1)$ & $15.6(14.9-17.1)$ & $11.4(8.1-11.5)$ & $30.8(19.4-50.5)$ \\
\hline$p$ value & 0.484 & 0.652 & 0.594 & 1.000 & 0.967 \\
\hline
\end{tabular}

Data are median values. Numbers in parentheses indicate interquartile range. $p$ values were calculated using Mann-Whitney test. ${ }^{*} p<0.05$

Among the PCT parameters, MTT $_{\text {CT }}$ showed significant differences according to the histologic types (PCC or non-PCC), T-staging ( $\leq \mathrm{T} 2$ vs. $\geq \mathrm{T} 3$ ), $\mathrm{N}$-staging (NO vs. $\mathrm{N}$-positive), and the expression level of EGFR ( $\leq$ faint vs. $\geq$ moderate staining) $(p<0.05)$ (Table 5); none of the PCT parameters showed significant differences according to the tumor differentiation $(p>0.05)$.

\section{DISCUSSION}

Our study results demonstrated the feasibility of CEUS to quantify the tumor perfusion of gastric cancers with a high success rate $(88.4 \%, 38 / 43)$. Gastric cancers have been regarded as non-suitable targets for CEUS as lesions are frequently obscured by bowel gas on ultrasonography and can be highly affected by the patients' respiratory motion. However, these limitations have been substantially overcome by double contrast-enhanced technique for data acquisition (15) and motion-correction algorithms for data post-processing (19) which were used in our study. Considering that perfusion analysis of gastric cancers is gaining increasing attention in the preoperative assessment as well as monitoring of treatment response in patients with gastric cancer $(13,14)$, CEUS is a promising tool that can be used as a non-invasive approach without concerns of ionizing radiation or renal dysfunction (24). However, it should be noted that perfusion quantification through CEUS can be applied in select patients with visible lesions on gray-scale images using the double contrast-enhanced technique and gastric cancers of the lower location in the stomach as indicated by the technical success rate attained in our study.

Studies to assess the perfusion of gastric cancers have mainly focused on PCT than CEUS $(12,23,25)$ but CEUS has recently shown promising results (26). However, the interchangeability or differences between the parameters 

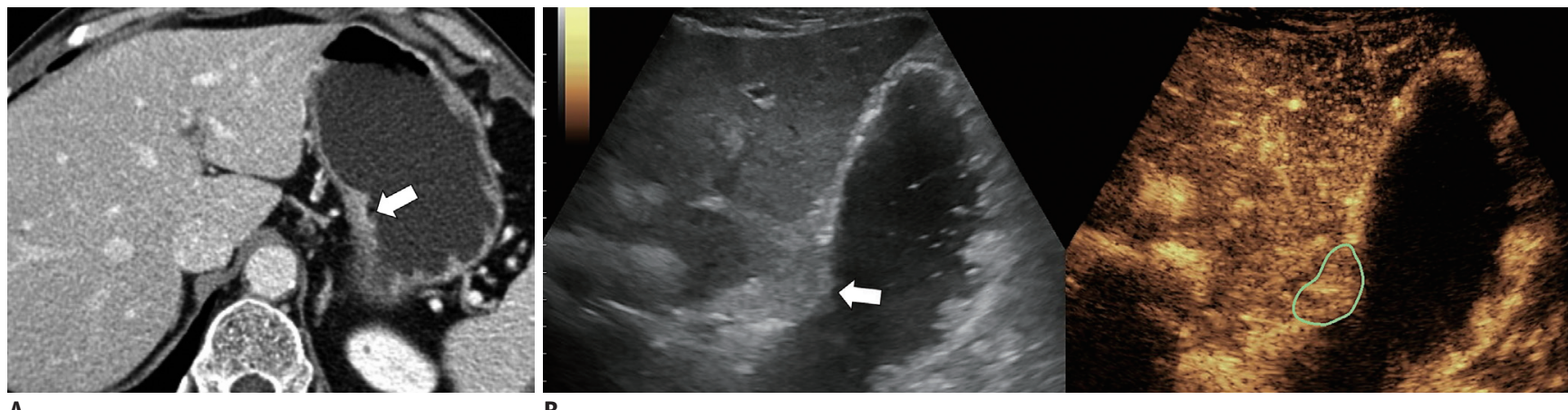

A

B

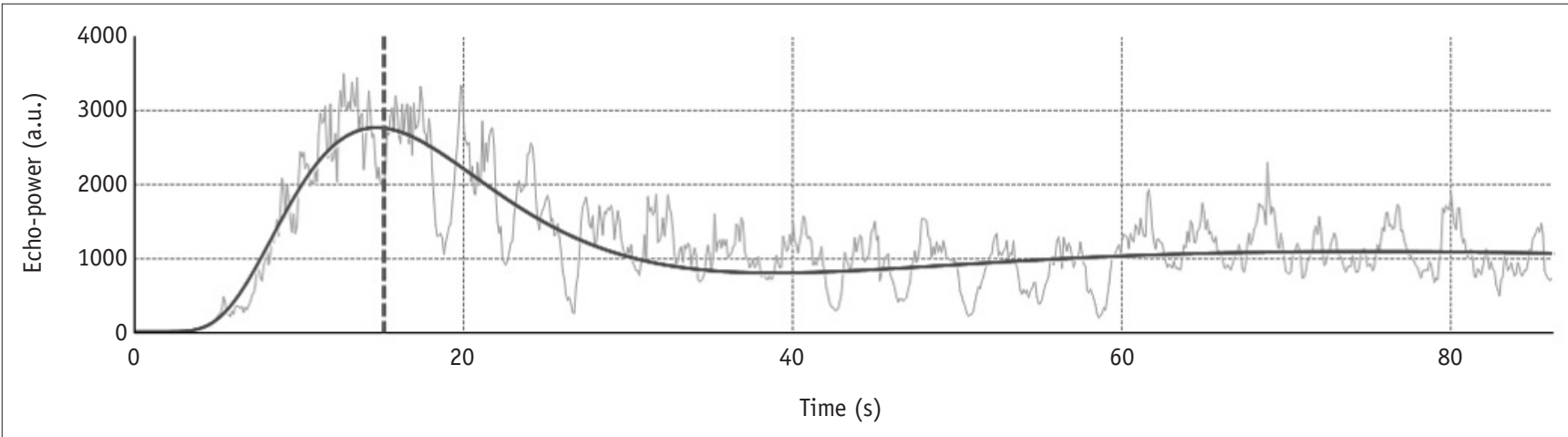

Fig. 2. Sixty-two-year-old male patient with surgically-proven pT2NO gastric cancer of moderately-differentiated adenocarcinoma. A. Preoperative contrast-enhanced CT image shows enhancing ulceroinfiltrative mass (arrow) at level of gastric mid body. B. CEUS image shows enhancing mass (arrow, green ROI) at corresponding location of water-distended stomach. C. Time-intensity curve analysis reveals PI of 2619 a.u. and AUC of 48820 a.u. ROI = region of interest

derived from CEUS and PCT remain unknown. In this study, assessment of the relationship between the CEUS and PCT parameters of gastric cancers revealed that time-related parameters of RT and TTP US of CEUS showed significant positive correlations with TTP $\mathrm{P}_{\mathrm{CT}}$ of PCT, while PI, AUC, and $\mathrm{MTT}_{\text {US }}$ of CEUS showed no significant correlations. These results are in agreement with those of previous studies on liver tumors which demonstrated the correlations between the arterial perfusion parameters of CEUS and PCT but not that of the portal perfusion parameters $(27,28)$. RT and TTP ${ }_{\mathrm{US}}$ as well as $T_{T P}$ are considered to reflect tissue vascularity and hence show high values in tumors with high vascularity and low values in tumors with low vascularity. In contrast, MTT $_{\text {US }}$ and $\mathrm{MTT}_{\mathrm{CT}}$ were not correlated in our study possibly due to the use of different contrast agents of different enhancement kinetics between the modalities (pure intravascular agent of CEUS and diffusible agent of PCT). Considering that MTT indicates the average time taken by blood to pass through the capillary network, higher tumor vascularity and arteriovenous shunts may shorten the MTT $_{\text {US }}$ as well as the $\mathrm{MTT}_{\mathrm{CT}}(6)$; whereas, the leaky vasculature of the tumor would prolong the $\mathrm{MTT}_{\mathrm{CT}}$ and have no effect on the MTT $\mathrm{MS}_{\text {(29) }}$ as ultrasound contrast agents do not leak into the interstitial space. This difference may explain the result of our study of poor correlation between the $\mathrm{MTT}_{\mathrm{CT}}$ and $\mathrm{MTT}_{\text {US }}$. These results suggest that CEUS and PCT are not interchangeable in the perfusion quantification of gastric cancers.

In our study, the comparison of perfusion parameters according to the histologic features of gastric cancers indicated that the amplitude-related parameters of PI and AUC of CEUS showed significantly higher values in WD or MD cancers than in PD cancers, while none of the PCT parameters showed significant differences according to tumor differentiation. PI and AUC of CEUS are indicators of the degree of enhancement and reflect the vascularity of gastric cancers (20) as well as other tumor types such as colorectal, ovarian, and prostate cancers (30-32). Our study results of lower PI and AUC values for the PD tumor type are in agreement with those of previous studies of lower density of the microvessel based on histology (33) and lower BF on PCT (34) in PD gastric cancers. Nevertheless, several previous studies have demonstrated that PD gastric cancers show higher microvessel density (35) or higher values of perfusion imaging parameters (25) than WD or MD tumors. 


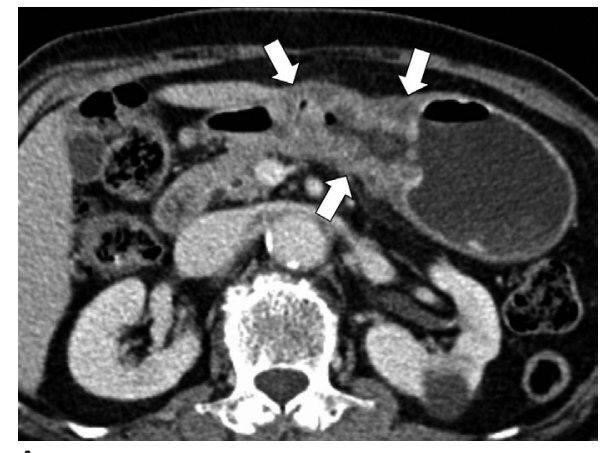

A

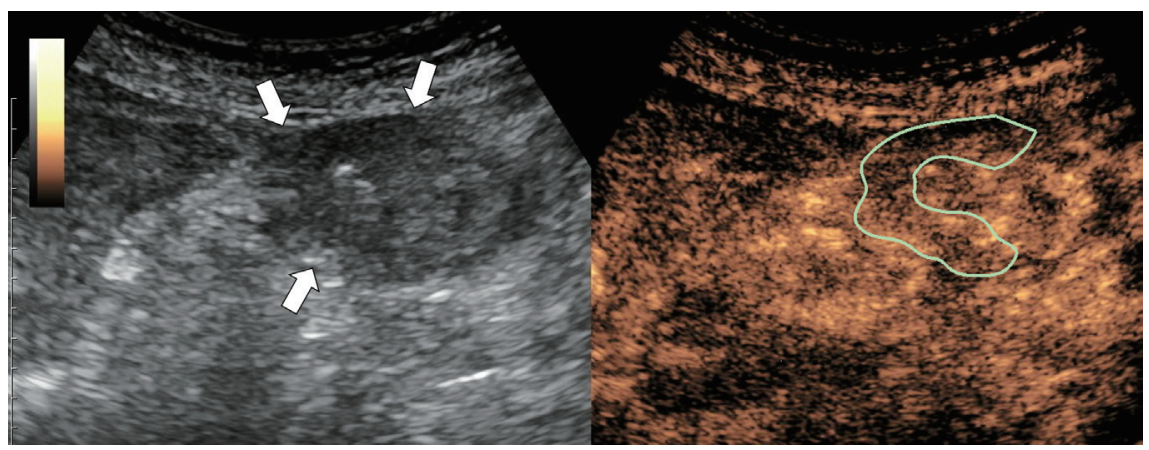

B

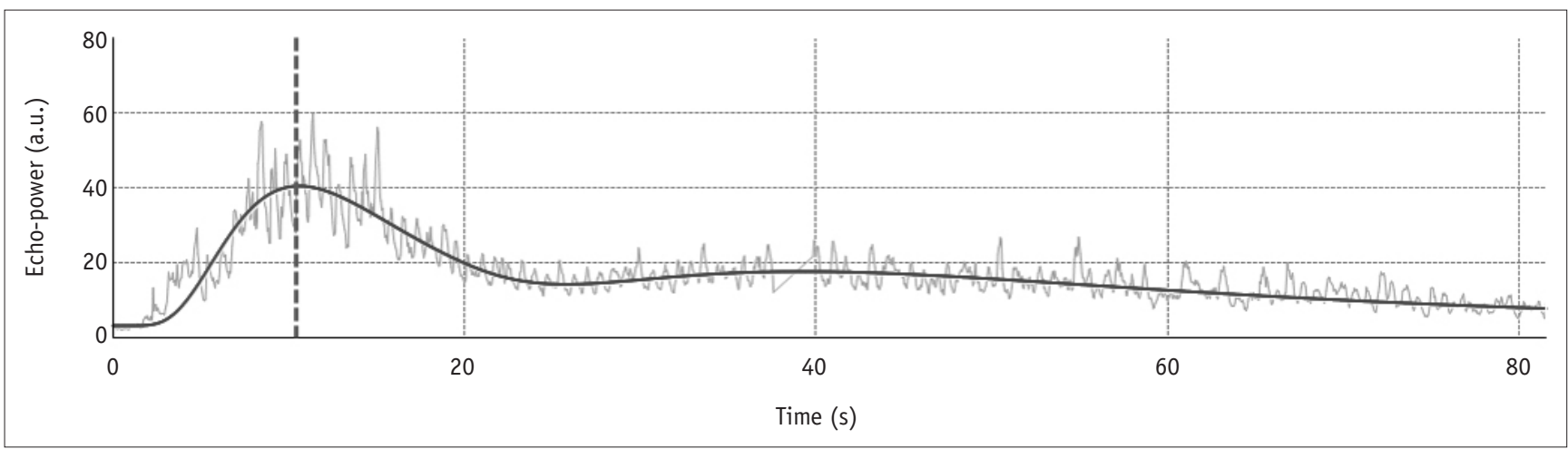

C

Fig. 3. Eighty-two-year-old female patient with surgically-proven pT3NO gastric cancer of poorly-differentiated adenocarcinoma. A. Axial CT image shows ulceroinfiltrative mass involving gastric low body and antrum (arrows). B. CEUS image reveals mass (arrows, green ROI) showing mild enhancement. C. Time-intensity curve analysis reveals PI of 36.9 a.u. and AUC of 342.0 a.u.

To clarify the relationship between the CEUS parameters and histologic features, further studies including multivariate analysis of a larger study population to control confounding variables are needed. With regard to PCT, in our study, the $\mathrm{MTT}_{\text {CT }}$ showed higher values in the tumors of more advanced T and N-stage and PCC type, and lower values in the tumors with higher EGFR expression, while none of the CEUS parameters showed significant differences according to these histologic features. Therefore, our study results suggested that the combined CEUS and PCT parameters would provide more detailed characteristics of the tumor perfusion in gastric cancer patients.

Our study has some limitations. First, our study population was relatively small and the reference standard for histologic features was obtained in only a subgroup of patients who underwent gastrectomy. Second, we performed two-dimensional perfusion analysis of CEUS which has capability to provide information of a single slice of the tumor. Moreover, although we selected an image plane containing the largest tumor dimension, the image plane for CEUS may not have been identical to that for PCT; to overcome this limitation, volumetric analysis of the tumor perfusion is a promising tool to accurately reflect the heterogeneity of the whole tumor. Third, our study indicated that some CEUS parameters may be useful to predict the pathologic prognostic features of gastric cancers, however, it did not directly assess the predictive values of the patients' outcomes. Further prospective studies with longterm follow-up are required to determine the prognostic function of CEUS in patients with gastric cancer.

In conclusion, CEUS is technically feasible for quantification of the tumor perfusion in patients with gastric cancers and provides correlative and complementary information to that through PCT to enable predicting histologic features.

\section{Conflicts of Interest}

The authors have no potential conflicts of interest to disclose.

\section{Acknowledgments}

We thank Chris Woo, BA, for his assistance in editing the manuscript. 


\section{ORCID iDs}

\author{
Se Hyung Kim \\ https://orcid.org/0000-0001-8664-0356 \\ Ijin Joo \\ https://orcid.org/0000-0002-1341-4072 \\ Dong Ho Lee \\ https://orcid.org/0000-0001-8983-851X \\ Joon Koo Han \\ https://orcid.org/0000-0001-5916-5545
}

\section{REFERENCES}

1. Soerjomataram I, Lortet-Tieulent J, Parkin DM, Ferlay J, Mathers C, Forman D, et al. Global burden of cancer in 2008: a systematic analysis of disability-adjusted life-years in 12 world regions. Lancet 2012;380:1840-1850

2. De Vita F, Giuliani F, Galizia G, Belli C, Aurilio G, Santabarbara $\mathrm{G}$, et al. Neo-adjuvant and adjuvant chemotherapy of gastric cancer. Ann Oncol 2007;18 Suppl 6:vi120-vi123

3. Shah MA, Ajani JA. Gastric cancer--an enigmatic and heterogeneous disease. JAMA 2010;303:1753-1754

4. Garcia-Figueiras R, Goh VJ, Padhani AR, Baleato-Gonzalez S, Garrido M, Leon L, et al. CT perfusion in oncologic imaging: a useful tool? AJR Am J Roentgenol 2013;200:8-19

5. Padhani AR. Dynamic contrast-enhanced MRI in clinical oncology: current status and future directions. J Magn Reson Imaging 2002;16:407-422

6. Kim SH, Kamaya A, Willmann JK. CT perfusion of the liver: principles and applications in oncology. Radiology 2014;272:322-344

7. O'Connor JP, Jackson A, Parker GJ, Roberts C, Jayson GC. Dynamic contrast-enhanced MRI in clinical trials of antivascular therapies. Nat Rev Clin Oncol 2012;9:167-177

8. Frohlich E, Muller R, Cui XW, Schreiber-Dietrich D, Dietrich CF. Dynamic contrast-enhanced ultrasound for quantification of tissue perfusion. J Ultrasound Med 2015;34:179-196

9. Jahng GH, Li KL, Ostergaard L, Calamante F. Perfusion magnetic resonance imaging: a comprehensive update on principles and techniques. Korean J Radiol 2014;15:554-577

10. Lu Q, Huang BJ, Wang WP, Li CX, Xue LY. Qualitative and quantitative analysis with contrast-enhanced ultrasonography: diagnosis value in hypoechoic renal angiomyolipoma. Korean J Radiol 2015;16:334-341

11. Lv WF, Han JK, Cheng DL, Zhou CZ, Ni M, Lu D. CT perfusion imaging can predict patients' survival and early response to transarterial chemo-lipiodol infusion for liver metastases from colorectal cancers. Korean J Radiol 2015;16:810-820

12. Zhang H, Pan Z, Du L, Yan C, Ding B, Song Q, et al. Advanced gastric cancer and perfusion imaging using a multidetector row computed tomography: correlation with prognostic determinants. Korean J Radiol 2008;9:119-127

13. Lee DH, Kim SH, Im SA, Oh DY, Kim TY, Han JK.
Multiparametric fully-integrated 18-FDG PET/MRI of advanced gastric cancer for prediction of chemotherapy response: a preliminary study. Eur Radiol 2016;26:2771-2778

14. Joo I, Lee JM, Han JK, Yang HK, Lee HJ, Choi BI. Dynamic contrast-enhanced MRI of gastric cancer: correlation of the perfusion parameters with pathological prognostic factors. J Magn Reson Imaging 2015;41:1608-1614

15. Li T, Lu M, Song J, Wu P, Cheng X, Zhang Z. Improvement to ultrasonographical differential diagnosis of gastric lesions: the value of contrast enhanced sonography with gastric distention. PLoS One 2017;12:e0182332

16. Xue H, Ge HY, Miao LY, Wang SM, Zhao B, Wang JR, et al. Differential diagnosis of gastric cancer and gastritis: the role of contrast-enhanced ultrasound (CEUS). Abdom Radiol (NY) 2017;42:802-809

17. Yan C, Bao X, Shentu W, Chen J, Liu C, Ye Q, et al. Preoperative gross classification of gastric adenocarcinoma: comparison of double contrast-enhanced ultrasound and multi-detector row CT. Ultrasound Med Biol 2016;42:14311440

18. Li S, Huang P, Wang Z, Chen J, Xu H, Wang L, et al. Preoperative $T$ staging of advanced gastric cancer using double contrast-enhanced ultrasound. Ultraschall Med 2012;33:E218-E224

19. Tranquart F, Mercier L, Frinking P, Gaud E, Arditi M. Perfusion quantification in contrast-enhanced ultrasound (CEUS)--ready for research projects and routine clinical use. Ultraschall Med 2012;33 Suppl 1:S31-S38

20. Shiyan L, Pintong $H$, Zongmin W, Fuguang $H$, Zhiqiang $Z$, Yan $Y$, et al. The relationship between enhanced intensity and microvessel density of gastric carcinoma using double contrast-enhanced ultrasonography. Ultrasound Med Biol 2009;35:1086-1091

21. Ang J, Hu L, Huang PT, Wu JX, Huang LN, Cao CH, et al. Contrast-enhanced ultrasonography assessment of gastric cancer response to neoadjuvant chemotherapy. World J Gastroenterol 2012;18:7026-7032

22. Ranganath PG, Robbin ML, Back SJ, Grant EG, Fetzer DT. Practical advantages of contrast-enhanced ultrasound in abdominopelvic radiology. Abdom Radiol (NY) 2018;43:9981012

23. Lee DH, Kim SH, Joo I, Han JK. CT Perfusion evaluation of gastric cancer: correlation with histologic type. Eur Radiol 2018;28:487-495

24. Jang JY, Kim MY, Jeong SW, Kim TY, Kim SU, Lee SH, et al. Current consensus and guidelines of contrast enhanced ultrasound for the characterization of focal liver lesions. Clin Mol Hepatol 2013;19:1-16

25. Zongqiong S, Xiaohong L, Wei C, Jiangfeng Z, Yuxi G, Zhihui $X$, et al. CT perfusion imaging of the stomach: a quantitative analysis according to different degrees of adenocarcinoma cell differentiation. Clin Imaging 2016;40:558-562

26. Lang SA, Moser C, Gehmert S, Pfister K, Hackl C, Schnitzbauer $A A$, et al. Contrast-enhanced ultrasound (CEUS) detects 
effects of vascular disrupting therapy in an experimental model of gastric cancer. Clin Hemorheol Microcirc 2014;56:287299

27. Goetti R, Reiner CS, Knuth A, Klotz E, Stenner F, Samaras P, et al. Quantitative perfusion analysis of malignant liver tumors: dynamic computed tomography and contrast-enhanced ultrasound. Invest Radiol 2012;47:18-24

28. Meijerink MR, van Waesberghe JH, van Schaik C, Boven E, van der Veldt AA, van den Tol $P$, et al. Perfusion CT and US of colorectal cancer liver metastases: a correlative study of two dynamic imaging modalities. Ultrasound Med Biol 2010;36:1626-1636

29. Zhou JH, Zheng W, Cao LH, Liu M, Luo RZ, Han F, et al. Quantitative evaluation of viable tissue perfusion changes with contrast-enhanced greyscale ultrasound in a mouse hepatoma model following treatment with different doses of thalidomide. Br J Radiol 2011;84:826-832

30. Zhuang H, Yang ZG, Chen HJ, Peng YL, Li L. Time-intensity curve parameters in colorectal tumours measured using double contrast-enhanced ultrasound: correlations with tumour angiogenesis. Colorectal Dis 2012;14:181-187
31. Wang J, Lv F, Fei X, Cui Q, Wang L, Gao X, et al. Study on the characteristics of contrast-enhanced ultrasound and its utility in assessing the microvessel density in ovarian tumors or tumor-like lesions. Int J Biol Sci 2011;7:600-606

32. Yang JC, Tang J, Li Y, Fei X, Shi H. Contrast-enhanced transrectal ultrasound for assessing vascularization of hypoechoic BPH nodules in the transition and peripheral zones: comparison with pathological examination. Ultrasound Med Biol 2008;34:1758-1764

33. Kawamura M, Naganuma H, Shibuya R, Kikuchi T, Sakai Y, Nagasaki $F$, et al. Analysis of microvascular density in early gastric carcinoma using magnifying endoscopy with narrowband imaging. Endosc Int Open 2016;4:E832-E837

34. Satoh A, Shuto K, Okazumi S, Ohira G, Natsume T, Hayano K, et al. Role of perfusion CT in assessing tumor blood flow and malignancy level of gastric cancer. Dig Surg 2010;27:253-260

35. Tenderenda M, Rutkowski P, Jesionek-Kupnicka D, Kubiak R. Expression of CD34 in gastric cancer and its correlation with histology, stage, proliferation activity, p53 expression and apoptotic index. Pathol Oncol Res 2001;7:129-134 